Instituto Internacional de Investigación y Desarrollo Tecnológico Educativo INDTEC, C.A.

DOI: https://doi.org/10.29394/scientific.issn.2542-2987.2017.2.6.17.322-336

OAI-PMH: http://www.indteca.com/ojs/index.php/Revista Scientific/oai

\title{
La Gerencia Avanzada en el Proceso de Investigación Científica Universitaria
}

\author{
Autor: Carlos Argimiro Figueredo Álvarez \\ Universidad Centroccidental Lisandro Alvarado, UCLA \\ carlosfigal@gmail.com; calvarez@ucla.edu.ve \\ Lara, Venezuela
}

\section{Resumen}

Es propósito de este ensayo, luego del análisis de las ideas propuestas por diferentes autores mencionados a lo largo del texto, es presentar a la comunidad científica e interesados en la temática, reflexiones sobre el desempeño cotidiano de los investigadores universitarios y su visión gerencial. Los investigadores no sólo deben fortalecer sus competencias en lo metodológico, también es necesario la formación y actuación con pensamiento gerencial, en la medida que haya una aceptación y comprensión del proceso investigativo como una acción gerencial, en esa misma medida la respuesta a la sociedad será mucho más acertada y pertinente, toda vez que se habrán conjugado diferentes e importantes fortalezas que en efecto sinérgico, harán del acto investigativo y sus resultados una categoría fenomenológica y social de avanzada.

Palabras clave: formación de investigadores; investigador científico; administración de la investigación. 


\title{
Advanced Management in the University Scientific Research Process
}

\begin{abstract}
It is the purpose of this essay, after the analysis of the ideas proposed by different authors mentioned throughout the text, is to present to the scientific community and interested in the thematic, reflections on the daily performance of university researchers and their managerial vision. Researchers should not only strengthen their methodological competences, but also the training and performance with management thought, to the extent that there is an acceptance and understanding of the investigative process as a managerial action, to that same measure the response to society will be much more accurate and pertinent, since they will have combined different and important strengths that synergistic effect, will make the investigative act and its results an advanced phenomenological and social category.
\end{abstract}

Keywords: training of researchers; scientific researcher; administration of research. 


\section{Aspectos Introductorios}

El cumplimiento del compromiso social por parte de las universidades venezolanas, el cual adquieren como razón de su creación y existencia, se manifiesta de múltiples maneras a saber: cantidad, diversidad, calidad técnica, científica y ciudadana de sus egresados, diversidad de carreras y menciones tanto en el pregrado como en el postgrado, ejecución de proyectos de intervención orientados a satisfacer necesidades sociales, generación de espacios para la participación, discusión y aportes sobre los diferentes problemas sociales, culturales, técnicos y científicos; ejecución y difusión de investigaciones dirigidas a solución de problemas o aportes para el desarrollo científico, creación y actualización de conocimientos y saberes, entre otras.

Las universidades reciben y soportan diferentes presiones y exigencias, unas generadas por la particular manera de entender y satisfacer su compromiso social, el cual debe cumplirse en un contexto complejo, competitivo y altamente tecnologizado, donde se reconoce la importancia de lo intangible, de profundas e importantes incertidumbres y vertiginosos cambios de paradigmas, otras, generadas por la visión y expectativa que tiene la sociedad sobre el rol de la universidad y por último, las generadas por las expectativas gubernamentales sobre su praxis cotidiana, su comportamiento ideológico y sus ejecuciones presupuestarias. Estas presiones y exigencias las obligan de alguna manera a repensar la razón de su existencia y a revisar sus esquemas, estilos y estrategias gerenciales aplicados en sus funciones básicas (Docencia, investigación, extensión y fomento).Todo esto, según Ferrer y Pelekais (2004a), hace más compleja la gestión universitaria y compromete la calidad de sus respuestas en cuanto a su oportunidad y eficiencia, pero aun así, según Soto (1998), citado en Ferrer y Pelekais, (2004b), la universidad venezolana ha aportado al desarrollo económico del país e históricamente ha respondido impulsando el desarrollo del conocimiento de diferentes generaciones. 
Especial importancia en esta coyuntura adquiere el docente en su desempeño como investigador, como el agente social con mayor responsabilidad en la materialización hacia lo interno y visibilización hacia lo externo del cientificismo y de la función investigación atribuida a las universidades, es responsable además de la producción y divulgación del conocimiento y desarrollo de la ciencia, por eso debe asumir la realización de sus investigaciones no sólo como un problema epistemológico y metodológico, sino, según Padrón (2005), asumirlo también como un problema de orden gerencial u organizacional; es decir, un proceso de creación gerencial, el cual articulado con las otras funciones y responsabilidades universitarias se traduce a su vez en un activador de fuerzas y razones organizacionales orientadas a la concreción y asunción del liderazgo social que corresponde a la universidad en cuanto a la producción, difusión y legitimación social del conocimiento y el avance de la ciencia.

Al asumir la investigación como un proceso de orden gerencial, surgen las interrogantes de cuales fundamentos gerenciales teóricos y praxeológicos deben integrarse en la función investigación universitaria y cuáles características, atributos o valores identificarán o permitirán identificar la competencia gerencial de los investigadores

Por tanto, el propósito de este ensayo es resaltar y dar al investigador en su actuar como tal, el lugar de reconocimiento en el escenario investigativo, quien debe ser un permanente examinador y evaluador de sus propias fortalezas, habilidades, destrezas y debilidades, para en ese compromiso consigo mismo y con visión de estratega, pueda detectar y aplicar sus potencialidades en sus proyectos investigativos, es decir administrarse a sí mismo, autogerenciarse 
2. Visión organizativa de la investigación científica universitaria. La ciencia, el conocimiento y la gerencia; presencias vitales de las universidades.

Para el logro del funcionamiento sincronizado, interrelacionado y armónico, de las diferentes funciones universitarias y el logro del liderazgo institucional, las cualidades y competencias gerenciales que posean los investigadores son insumo de orden vital, lo que confirma Olivares (Citado en Machado, Reyes, Rietveldt, y Luquez, (2007), cuando señala que no existe una gestión coordinadora entre las funciones de la universidad por el hecho de algunos docentes carecer de la formación gerencial necesaria.

En la función investigación universitaria se distinguen tres vertientes o plataformas de enfoque, interconectadas, que se relacionan e interarticulan de manera permanente, a saber: la aplicada por la propia universidad a los aspectos administrativos de la investigación, la aplicada para la formación y estímulo de los investigadores (carrera del investigador) y por último la aplicada por el investigador directamente a la investigación, entendida ésta, como una concatenación de fases, procesos y actividades que se suceden y entrelazan de manera lógica y armónica y no sólo un mero ejercicio filosófico de reflexión y contemplación, por tanto es posible visualizar semejanzas con los procesos gerenciales productivos $u$ operativos de las organizaciones empresariales.

Los postulados teóricos gerenciales nacidos o emanados de estudios sobre empresas mercantiles fabriles, financieras y de servicios, han migrado y son aplicados en diferentes tipos de organizaciones, incluso en entornos de menor expresión colectiva, hasta individualizados, de allí que hoy se dialogue sobre la gerencia de la vida familiar, de la carrera profesional o laboral, de la gerencia de procesos, proyectos, funciones u operaciones organizacionales, y por supuesto, de gerencia de las universidades, instituciones que a decir de 
Monagas (2006), experimentan una turbulencia en sus esquemas organizativos.

El marco o ámbito legal de la acción universitaria, lo representa la Ley de Universidades (1970), en cuyo articulado la universidad es presentada como una comunidad de intereses espirituales cuya tarea es buscar la verdad y afianzar los valores trascendentales del hombre mediante la conjunción de profesores y estudiantes. Se espera de ella una función rectora en la educación, la cultura y la ciencia, materializada en la creación, asimilación y difusión del saber mediante la investigación y la enseñanza. En esta caracterización de la universidad venezolana, la investigación y la enseñanza (docencia) se destacan como sus funciones cimeras y su razón de ser.

Lo establecido en la mencionada ley, fue ratificado por las disposiciones de la Constitución de la República Bolivariana de Venezuela (2000), la cual en su artículo 109 establece, que es a través de la investigación científica, humanística y tecnológica como los profesores, estudiantes y egresados se dedicarán a la búsqueda del conocimiento, para el beneficio espiritual y material de la Nación.

Además de las disposiciones legales, es menester mencionar los diferentes acuerdos y pactos internacionales que Venezuela como signataria de organismos multilaterales, bloques regionales o sectoriales ha suscrito, de estos acuerdos también se desprenden para nuestro país y sus instituciones académicas, líneas de acción de obligatorio cumplimiento (Por ejemplo los acuerdos de la Organización de las Naciones Unidas para la Educación, la Ciencia y la Cultura-UNESCO), orientadas todas a posicionar lo científico académico en lugares de liderazgo y conducción social y a contribuir con las grandes soluciones que la humanidad reclama a la espera del bienestar colectivo.

Tal como lo señala Rojas (2006), en la comunidad científica y académica y en la sociedad en general, uno de los retos máximos de las 
organizaciones cualquiera sea su tipo y actividades, es la búsqueda del bienestar humano mediante la función gerencial. Así entonces, para la contribución al logro del bienestar humano, las universidades se valen de recursos físicos, contribución humana, actividades y procesos, componentes todos del proceso gerencial con el cual se gobierna la institución en toda su amplitud y complejidad.

Lo anterior permite indicar que la gerencia, la investigación, la ciencia y el conocimiento como sus productos, no representan fines en sí mismos, sólo son medios para el logro del bienestar humano,categorías conceptuales, entre las cuales se genera una interdependencia permanente, los elementos de uno son necesarios para el otro, así la gerencia se perfecciona por la investigación, la investigación se robustece con los procesos gerenciales, la ciencia identifica y modifica atributos de la gerencia y el conocimiento permite la aplicabilidad de la gerencia y de la ciencia, de esta manera los conceptos se entrelazan y direccionan a la universidad en el cumplimiento de sus fines sociales.

\section{El investigador universitario, necesidades epocales de formación gerencial.}

El investigador universitario tropieza con diferentes obstáculos representados por el factor tiempo, ausencia de una definición de carrera, baja atención presupuestaria y en su aspecto anímico quizás influye la relativamente escasa importancia dada internamente a la investigación tanto para el otorgamiento y cumplimiento de las dedicaciones como para la adjudicación de reconocimientos internos, además que en su desempeño convergen diferentes intereses, antagónicos o contrapuestos a veces, no siempre las propuestas investigativas individuales interesan a lo institucional o no se corresponden con sus metas o planes.

En el sector universitario las autoridades nacionales también intervienen, generando, proponiendo o estableciendo áreas prioritarias de 
investigación no siempre coincidentes con las expectativas de la universidad $\mathrm{y}$ las de los investigadores, por tanto la universidad y los docentes universitarios conviven en un permanente dilema entre la autonomía para la búsqueda de la verdad y la prestación de servicios al Estado venezolano, a lo que se agrega la visión o percepción individualista o colectivizada que se tenga sobre la universidad.

\section{De la administración tradicional a la gerencia avanzada en la investigación científica.}

Resultante del análisis a diferentes definiciones del concepto, puede decirse entonces que gerencia avanzada es un concepto en plena construcción, se explica como un paradigma emergente que intenta hacer entender la direccionalidad en gestión de las organizaciones y sus procesos, cualquiera sea su tipo y objeto, y además, la forma de definirlos y estudiarlos, en consonancia con las exigencias de la sociedad de la información y del conocimiento, lo cual implica mantenerse, según Gutiérrez (2005), al ritmo de los cambios de los nuevos paradigmas, por tanto, no es una expresión remozada de la gerencia tradicional, es un estadio superior.

Gerencia avanzada es un concepto totalmente aplicable al proceso de investigación científica en tanto este se asuma como un asunto gerencial y significaría la aprehensión y aplicación de una serie de elementos y atributos, los cuales, a los efectos analíticos en este ensayo, se presentan separados, pero en la dinámica gerencial, cualquiera sea su ámbito, conforman una totalidad interconectada, a saber:

Multidimensionalidad: significa que el gerente debe estar atento a que la organización o el proceso del cual es responsable, sea una suma de elementos provenientes de diferentes niveles humanos, materiales, intangibles, cada uno con su esencialidad, con sus diferencias, que confluyen para un propósito común. 
Complejidad y transcomplejidad: el fundamento teórico y praxeológico de la gerencia, está impregnado de múltiples interacciones, relacionalidades y enfoques disciplinares. No hay un conocimiento específico que brinde o aporte todos los atributos que exhibe la gerencia y aprehende y aplica el gerente. La gerencia tanto en su discurso teorizante como en su práctica cotidiana, es una especie de crisol donde se forja un conocimiento puesto al servicio del colectivo y a la permanente búsqueda del bienestar humano como propósitos cimeros. La visión transcompleja de la gerencia se orienta entonces a una permanente observación y comprensión de las rupturas paradigmáticas y a las emergencias epistémicas.

Vocación de servicio: la gerencia como tal, tiene entre sus propósitos, solucionar problemas, dar respuesta oportuna, útil y veraz. No debe transformarse en una especie de muralla, donde se estrellen necesidades y expectativas. La vocación de servicios y de aportes solucionantes, es un valor gerencial asociado al de responsabilidad social, debe ser una práctica cotidiana.

Responsabilidad Social: la gerencia en su accionar debe generar un valor social agregado, más allá del mero cumplimiento de las metas organizacionales. Significa un compromiso, un pacto de servicio y utilidad, que trasciende y es plataforma para el reconocimiento social de la organización, sus procesos, productos y servicios, tanto hacia lo interno de la organización como hacia lo externo.

Producción de conocimiento: el ejercicio gerencial, independientemente de la organización o proceso del cual se trate, es una fuente permanente generadora de información y conocimiento, en la medida que la gerencia se autoexamine y sistematice los resultados obtenidos, contribuirá con el avance científico organizacional. En este sentido las organizaciones de carácter académico tienen una responsabilidad mayor. 
Inter y transdisciplinariedad: la gerencia no es un conocimiento aislado, es la suma de múltiples aportes científicos coincidentes en aspectos definidos. Las diferentes disciplinas aportantes para la construcción de la teoría gerencial, poseen aspectos comunes, que atraviesan en un imaginario recorrido horizontal, vertical y diagonal, sus estructuras.

Búsqueda de bienestar humano: en el encuentro social, se mantiene de manera recurrente una interacción bidireccional, de lo individual a lo colectivo y viceversa. El individuo aporta a la sociedad y ésta, aporta al individuo. Esta reciprocidad tiene en el accionar gerencial un mecanismo u oportunidad de hacer más expedito este intercambio de beneficios. El individuo con su aporte laboral, científico, ciudadano contribuye a la conformación de una sociedad con mejores índices de calidad de vida, más amigable, humana, y apropiada para su desarrollo y realización

Contextualización epocal: la gerencia, para ser útil y con productividad, debe estar atenta a los significados, signos y mensajes epocales, de no hacerlo se estaría frente a una gerencia ortodoxa, tradicional, desubicada, jamás de avanzada, que por desconocimiento e impracticidad, no podría aprovechar y aplicar todas las potencialidades que pudiera poseer.

Orientación a la calidad: de la gerencia se espera solución de problemas significativos, de interés o repercusión colectiva, administración idónea de recursos, integración de talento humano, en términos de prontitud, accesibilidad y oportunidad.

Legitimación y visibilidad social: la gerencia existe en tanto esté en función de intereses colectivos y superiores. Debe procurar impactar el entorno social mediante su ejecutoria, independientemente de las dimensiones organizacionales donde se desempeñe. El reconocimiento y legitimación social serán indicadores de su eficiencia. Es una dimensión asociada directamente a la responsabilidad social. 
Dinamismo e innovación: la gerencia innovadora y dinámica, puede transformarse en el motor que induce los cambios sociales, organizacionales y científicos. La cultura de la innovación como valor organizacional e individual debe ser comprendida y asumida como una manifestación esencial y significado epocal de la sociedad del conocimiento y medio o cauce para el desarrollo y reforzamiento de las potencialidades creadoras.

Conciencia planetaria (compromiso con la vida); el aprovechamiento de las bondades de la naturaleza, el progreso de la humanidad y sus organizaciones, no debe significar un sacrificio para las generaciones del presente ni un panorama sombrío para las generaciones del futuro. La gerencia, incluso por una mera razón de sobrevivencia debe tratar con respeto al medio natural. En una visión compleja del fenómeno de la gerencialidad, sus actores y agentes deberán estar atentos a los impactos y sus efectos que los productos organizacionales causen. La conciencia planetaria se inscribe en el emergente paradigma de la educación holista, que pretende dar respuesta a las cambiantes y cada vez más complejas necesidades sociales.

Prospectividad: el humano crea las organizaciones con propósito de trascendencia y en ese afán de trascender, ejercita su capacidad de influir en la construcción del futuro, se anticipa, genera condiciones, crea escenarios que permitan a sus organizaciones y a si mismo robustecer su pensamiento estratégico y estimular y fortalecer su aprendizaje.

Amplitud: los humanos son diferentes entre sí, tanto en el plano físico como en el de las acciones intelectivas. Todas las organizaciones y cada uno de sus procesos son diferentes La gerencia debe ser consciente de la diversidad, Por tanto, no habrá una fórmula única para gerenciar, tampoco una postura profesionista, las circunstancias también determinan las reacciones y acciones.

Conocimiento del entorno: el entorno no es algo estático, inmutable, tampoco es un plácido espacio físico, lo conforman condiciones climáticas, 
costumbres, valores, prácticas, legislaciones, historia, creencias. Un conocimiento profundo de lo que la rodea, reviste garantías razonables de éxito para la gerencia.

Aptitud positiva ante la incertidumbre: la falta de certeza es inmanente a la acción humana, por ende, a la organizacional y sus procesos. No siempre se tendrá toda la información que se requiere para la toma de alguna decisión. El gerente consciente, ante la incertidumbre no se arredra ni amilana. La gerencia debe mantener equilibrio entre la información que se posee y la que se considera, carece.

Formación de ciudadanía: dentro del área de su incumbencia, la gerencia tiene la posibilidad de participar en la formación de ciudadanía mediante actividades y estrategias que potencien los valores ciudadanos, el interés por los asuntos sociales, desde lo interno de la organización hasta irradiar en el entorno social inmediato. Incluso por esta vía, la propia organización estaría propiciando la construcción de un clima organizacional ideal.

Claridad metodológica: por ser una disciplina científica, la gerencia, tanto para su estudio como para su práctica, se apoya en teorías demostradas. Como proceso, la gerencia posee sus métodos, por medio de los cuales, los actores gerenciales obtienen sus mejores resultados. Cada modalidad de gerencia, activará su propio método.

Pensamiento gerencial: aunque parezca tautológico, quien asuma la responsabilidad de gerenciar, debe ser, parecer, pensar y actuar como gerente. Debe acercarse a los asuntos de su incumbencia con visión estratégica y amplitud de pensamiento. En la aptitud, formación y metodología gerencial estriba grandemente buena parte del éxito gerencial.

\section{Reflexiones finales}

Como concepto epistémico, la gerencia avanzada de la investigación 
universitaria, es un paradigma que irrumpe en el escenario académico universitario, intentando hacer entender la direccionalidad gestionaria de la función investigación cumplida y ejecutada por el propio investigador. Es una visión multidimensional y compleja, sustentada en valores y principios, dinámica, metodológicamente innovadora, socialmente legítima, orientada a la producción, organización y visibilización del conocimiento, como una vía para lograr el bienestar humano mediante el cumplimiento de la responsabilidad social institucional y profesional, en un entorno cambiante e incierto.

Para ello, el investigador, como actor de esa visión, gestiona con pensamiento gerencial el proceso investigativo. Intenta también esta definición, saldar una especie de deuda, de anonimia, que generalmente ha existido alrededor del humano, quien como agente y actor de diferentes procesos sociales, es prácticamente borrado del imaginario social, oculto en las sombras de la ignorancia colectiva, se habla de la medicina y se omite al médico, de la ciencia y se omite al científico, se hace referencia a la educación y se ignora al educador, se habla de la investigación y se omite al investigador. Se menciona y da importancia a los procesos, pero son tratados como si en ellos no intervinieran personas, alrededor de quienes, precisamente giran, teóricamente, todos los objetivos.

\section{Referencias}

Ferrer, T. y Pelekais, C. (2004a,b). Tendencias Gerenciales y la Gestión Universitaria. Revista de Ciencias Sociales, Vol. X (1), pp. 148-163, FACES-LUZ. Recuperado de:

\section{http://www.redalyc.org/pdf/280/28010111.pdf}

Gutiérrez. L, (2005). Gerencia Avanzada: Un blanco cada vez más móvil.

Revista NEGOTIUM, Año 1, Nro. 2. Recuperado de: http://www.revistanegotium.org.ve/pdf/2/2art1.pdf 
Ley de Universidades (1970). Caracas, Venezuela. Gaceta Oficial № 1.429.

Extraordinario de fecha 8 de septiembre de 1970.

Machado, F, Reyes, L., Rietveldt, F. y Luquez, P. (2007). Articulación de las

Funciones Universitarias: Un Marco de Transformación

Académica. Revista Formación Gerencial, Año 6, Nro 6. Recuperado

de: http://produccioncientificaluz.org/index.php/rafg/article/view/808

Monagas, J. (2005). La Gerencia Universitaria ante el cambio institucional.

Revista Visión Gerencial. Recuperado de:

http://www.saber.ula.ve/bitstream/123456789/25094/2/articulo5.pdf

Padrón, J. (2005). Investigación, Universidad y Sociedad. II Seminario Internacional Educa. Ponencia. Marzo 2005. Recuperado de: http://padron.entretemas.com.ve/InvUnivSoc/InvestigacionUniversidad Sociedad.pdf

República Bolivariana de Venezuela (2000). Constitución de la República Bolivariana de Venezuela. Gaceta Oficial 5.453 del 24 de marzo de 2000. Segunda versión. Venezuela.

Rojas, L. (2006). Los retos de la Gerencia en la Sociedad de la Información. Conferencia Magistral correspondiente a la Clase Inaugural del Doctorado en Gerencia Avanzada 2da Cohorte Universidad Fermín Toro Revista Negotium. Año 2, Nro. 5, noviembre 2006, pp. 77-100. Recuperado de:

http://www.revistanegotium.org.ve/pdf/5/5Doc1.pdf 
Carlos Argimiro Figueredo Álvarez

e-mail: carlosfigal@gmail.com, calvarez@ucla.edu.ve

Nacido en Venezuela. Contador Público (UCLA), Especialista y Magister en Auditoria (UCLA), Doctorado en Finanzas y Contabilidad (DEA- UVA, España) Doctor en Gerencia Avanzada (UFT). Posdoctorado Estudios Libres (UFT). Docente Titular (UCLA). Depto. Contabilidad. Cátedra Contabilidad, Metodología de la Investigación. Docente postgrado en UCAT, LUZ, UNELLEZ, UFT, UCLA, ULA. Investigador acreditado en PEILA y PEll. Investigador en: Responsabilidad Social, Gobernabilidad Universitaria, Teoría Contable, Organizaciones Economía Social. Tutor y Jurado de trabajos de investigación en Pre y Postgrado. Arbitro y Autor de Artículos en Revistas Científicas. Jurado en Premios de Investigación, Ponente en eventos Científicos Regionales y Nacionales. Miembro ASOVAC.

El contenido de este manuscrito se difunde bajo una Licencia de Creative Commons ReconocimientoNoComercial-Compartirlgual 4.0 Internacional 